\title{
Perspective
}

\section{Innovation in Clinical Trials is Possible: The Hidden Opportunity in the Coronavirus Disease-2019 Emergency}

\author{
Valeria L. Scarano, PhD, MBA ${ }^{\prime *}$; Davide Smaldone, MBA ${ }^{2}$ \\ Clinical Study Manager, Medtronic, Rome, Italy \\ ${ }^{2}$ Corporate IT Demand Manager, Menarini Group, Florence, Itlay \\ *Corresponding author \\ Valeria L. Scarano, PhD, MBA \\ Clinical Study Manager, Medtronic, Rome, Italy; E-mail: valeria.scarano@embawe.sdabocconi.it
}

\section{Article information}

Received: June II th 2020; Accepted: July 16 ${ }^{\text {th }}, 2020$; Published: July 17 th 2020

\section{Cite this article}

ScaranoVL, Smaldone D. Innovation in clinical trials is possible:The hidden opportunity in the coronavirus disease-2019 emergency. Clin Trial Pract Open J. 2020; 3(I): 15-19. doi: 10.17|40/CTPOJ-3-113

\section{ABSTRACT}

The coronavirus disease-2019 (COVID-19) emergency has clearly shown the need to discover, test and produce a new vaccine but also in general the fragility of the current chain of clinical trials. Through this emergency, is it possible to look at the current clinical trial processes with new eyes? Is it possible to seize the opportunity for change and to have confidence in the possibility of their improvement? In this short article the authors wanted to give an optimistic answer to this question by pushing on the possibilities of innovation nowadays.

Keywords

Clinical trials; Innovation; Covid-19; Digital transformation.

\section{WHY?}

Tn the first months of this year we have well-known viruses and 1 hated them all; in particular, we hope that a vaccine against coronavirus disease-2019 (COVID-19) will be found, capable of recognizing and neutralizing it.

However, only a small fraction of the world population knows the actual timing for placing a vaccine on the market; and that in addition to the research and development phase, a clinical trial phase is required which takes the same amount of time dedicated to research and development, if not more.

The COVID-19 emergency not only brought out the need for a new vaccine but showed the extreme fragility of the current clinical trial chain, blocking or at best by postponing worldwide all clinical trials currently being under test for six months, over 51,000 studies being enrolled in May 2020. ${ }^{1}$ In last few months, access to hospitals has been banned for employees of pharmaceutical and biomedical companies and furthermore medical personnel had no time to dedicate to trials. ${ }^{2}$
The global clinical trial market size is expected to reach $\$$ 69.9 billion by 2027 , presenting a $5.1 \%$ compound annual growth rate (CAGR) during the forecast period. ${ }^{3}$ In addition, an increase in the volume and complexity of clinical trials has recently been observed, which play an important role in the research and development of new drugs and other products. Clinical trials have become increasingly capital intensive, increasing the overall cost of developing a drug.

Therefore, large and small pharmaceutical and biotechnology companies must be able to see in the COVID-19 emergency an opportunity for renewal, by rethinking in an innovative way the clinical trial processes, still highly depended by actions with a high human content, also considering that patient behavior and the demands of hospitals and doctors are evolving rapidly. ${ }^{4}$

So, while most people in the world today wonder when the COVID-19 vaccine will arrive, we try to reflect if more generally we can reduce the time to market of a vaccine, a drug or a medical device, and just in case how to do it. To take this step forward, companies need to show adaptability to overcome their work routine. 
Since the pandemic it is necessary to look at clinical trials process with different eyes. Yes, you can look at the problem from a different point of view even in very traditionalist areas, such as healthcare field. Even more in these moments of global difficulty, we must seize the opportunity for change, have confidence in the possibility of continuous improvement with the ambition to do something new, useful and great. It is necessary to find ways to challenge the status quo and indicate an alternative to this sector, a model that can also be sustainable in the future. ${ }^{5}$

But let's go back for a moment to the COVID-19 case, the time to market of the COVID-19 vaccine can vary from 12-months, according to the most optimistic forecasts, to 24-months, according to the most realistic ones. If for a clinical trial lasting 12-months on for example 10,000 patients we could save even 2 -months, does it sound good for you? 60-days compared to 360 may seem few, but if we think about contagious that occurred in 60-days or more simply to the world economy freeze for 60-days, perhaps our perspective would change.

Analyzing a drug or a biomedical device development process from several points of view, we are convinced that innovation can also be brought about by acting also on a phase different from research and development. You can innovate, you can also create value by expanding the data processing and delivery services and channels. Yes, because this vision will allow those who undertake this path to acquire much of the value of the clinical chain, in a large and growing market with an easily scalable model.

\section{WHAT?}

The crisis broke or cracked the existing links in the clinical chain or highlighted their weak links, including operations.

We believe that we are born to be connected, but is this also true for clinical trials? Is it possible to think a new fully connected clinical trial process? Can missing links be introduced to the clinical trial process which would allow to give it a fully connected circular structure?

Yes, we believe that an ally can be found in the isolation between the trial phases, undergone in last months, to perhaps discover that in the isolation (modularity) there is an added value for achieving the objectives.

It is important to work together to understand how many different ways existing to achieve the same goal. Then the confidence in the possibility of being able to remove the obstacles that will prevent each individual solution from fully expressing its potential, precisely this trust will do good for the business, for new businesses.

Is it possible to think of a solution that makes sponsor companies also process facilitators? Think about the use of real-time technologies as benefits carriers both to companies and to those who carry out clinical studies in hospitals? Is it possible to think the clinical study in a truly patient centric way? Could a simplification of processes improve the user experience for all involved actors?

However, for every idea that arises there have to be persons who build the infrastructure to turn it into reality. This infrastructure is what will make any change possible and success measurable. The strategic focus to implement this innovation will not be the introduction of a technological innovation but the use of already available technological solutions; essentially converting a technological innovation into what is called a value innovation.

The innovation of the process can reduce direct travel costs of company staff, management costs of studies in terms of data and safety management and research and development (R\&D) thanks to real-time data collection. It will also have a strong corporate, hospital and social benefit by reducing the duration of a clinical trial and therefore speeding up the product launch on the market. Many sectors need new value-cost frontiers, the health sector is certainly one of them.

There will be a profound change in the "old style" market of clinical trials, rethought with a new business model that will allow tangible benefits for all stakeholders. This innovation will allow existing markets to be recreated and expanded beyond the previous limits. With the regeneration of these markets and the expansion of boundaries, growth will take place because the innovative solution will drive new demand and convert non-consumers into consumers. The strength, weakness, opportunities, threats (SWOT) analysis implemented thinking of the introduction of innovation in clinical studies and shown in Figure 1 shows significant strengths and opportunities.

The global clinical trials market is extremely fragmented and for the most part managed directly by pharmaceutical or biomedical companies or partly entrusted to clinical consultancy companies, perhaps the fragmented nature of the actors involved has allowed the clinical trials sector to show itself so weak with the arrival of the pandemic from COVID-19. Today we have the opportunity to take charge of an infrastructure innovation for growth in communication and clinical connection. Mobility of people is a status in clinical trials, henceforth it is more data and information that will travel and fewer people.

If it is true that the virus has been a catalyst for some processes of change, accelerating (or rather, forcing) the adoption of methodologies such as smart working, on the other hand, however, it has only pushed the transposition into digital of the processes previously performed in office. This "digitization" is not and cannot be the digital transformation that companies need, but must represent the kick-off of a game, that of true digital transformation, which perhaps some companies still hoped would not have to play. ${ }^{6}$

In these weeks it has been clearly and painfully evident to everyone how basically all industries, besides maybe software 


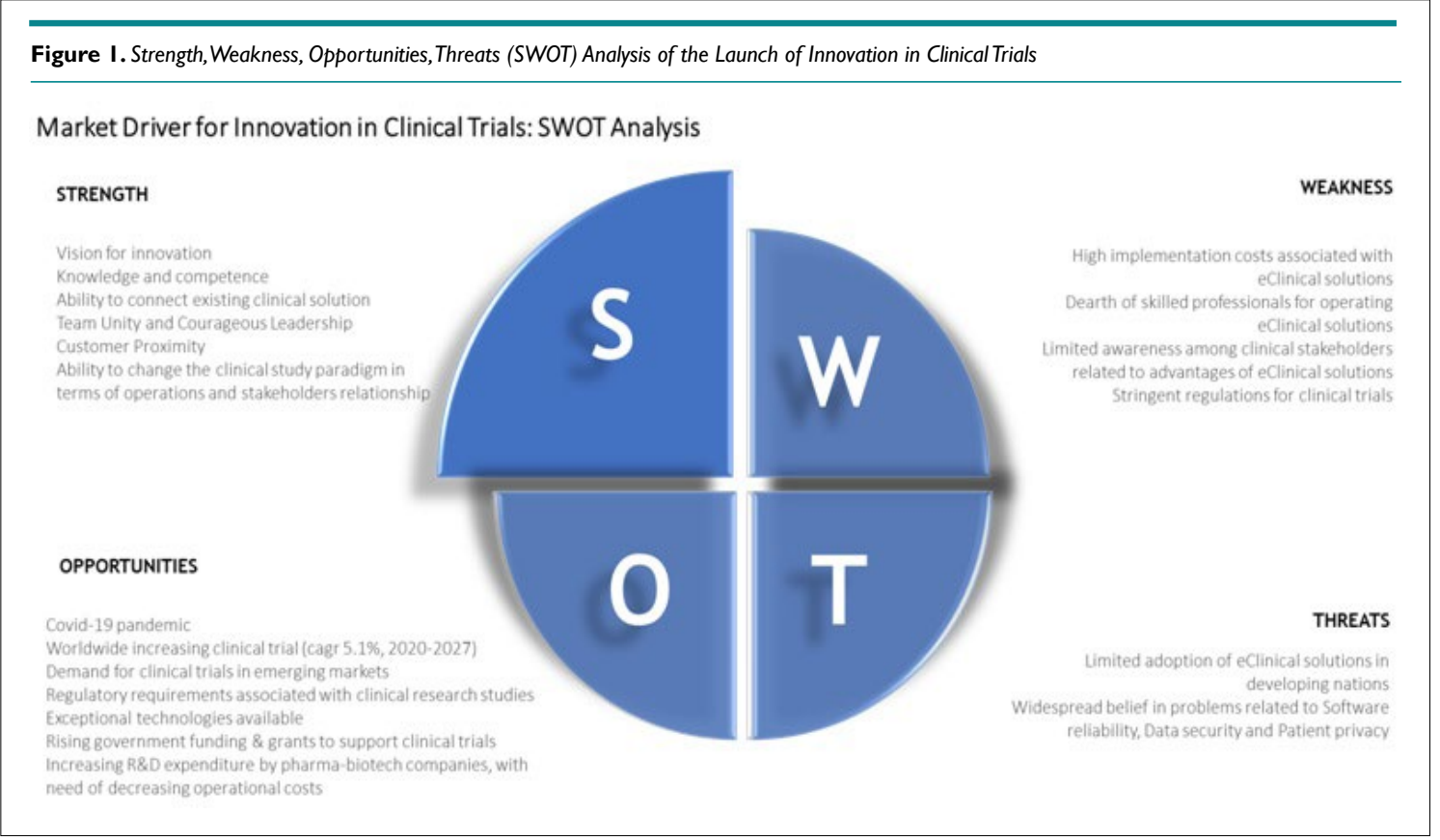

development, have been substantially unprepared to face such an event and this because they still mainly depend on face-to-face dynamics and on non-resilient processes.

Even in highly regulated environments such as pharmaceutical, biopharmaceutical and medical devices, R\&D and production it has been clear that, even though subject to highly documented processes managed through very strict and precise procedures, the dependency on the day-to-day physical contact was still one of the main drivers in the decision-making process of small, medium and large companies alike.

How could have pharmaceutical and biomedical companies dealt with the crisis and how resilient would they have been if processes such as clinical research had already undergone a true digital transformation? As we have said the tools to carry out this change are already available and in some cases the only action that needs truly to be performed is connecting the dots (dots, modules) that comprise a wider ecosystem where companies, contract research organization (CROs), hospitals and regulatory bodies live, share information and manage $\mathrm{R} \& \mathrm{D}$ processes.

Being disruptive is not strictly necessary, even though this topic is quite dear to many influencers in the digital \& innovation space, but simply using the various technologies that have been already studied and developed in different fields could suffice. ${ }^{7,8}$

Let's then start with dematerializing the documentation produced during clinical studies, a process that not only would save precious time for healthcare professionals that physically execute the study, but that would also improve data accuracy and integrity. In this scenario the relevant information would be input only once into the system and immediately checked before being transferred in a secure way to the following steps of the process. This approach is what is defined as data integrity by design, a concept that is particularly relevant in pharmaceutical production and that could be easily applied in clinical research with unchanged validity and efficacy. The electronic medial record (eMR) is undoubtedly the starting point to achieve this objective, even though it would need to be further improved in security and interoperability to be widely adopted. If a single eMR format could be used in all hospitals nationwide or at international level, this would represent a significant advantage for the tech companies working in this field that could start building solutions to manage and elaborate, in a totally secure and safe way, the information related to a specific patient instead of being forced to manage multiple eMR standards.

Technology now provides very effective solutions to ensure both the integrity and the secrecy of the data stored in a specific system both through traditional encryption tools as well as through innovative technologies such as blockchain. In a blockchain application, the same set of data is replicated simultaneously on the different nodes of the network that become more secured the more nodes are included in it (the so called "peripheral trust" effect). This tool could then contribute to improve data exchange reliability between different blockchain actors making at the same time the data flow completely transparent and fully traceable, ensuring data integrity.

Just only by mentioning two technologies it's possible to start imagining an ecosystem made of a set of information input only once at the source and then securely and transparently managed on-line. These tool could be always available to the people that need to use it and could be developed using a format that would foster data exchange and enrichment through the various steps of the clinical process.

The use of artificial intelligence (AI) and machine learning applied to this data could help selecting in a quick and efficient way the most suitable patients for a specific clinal study that 


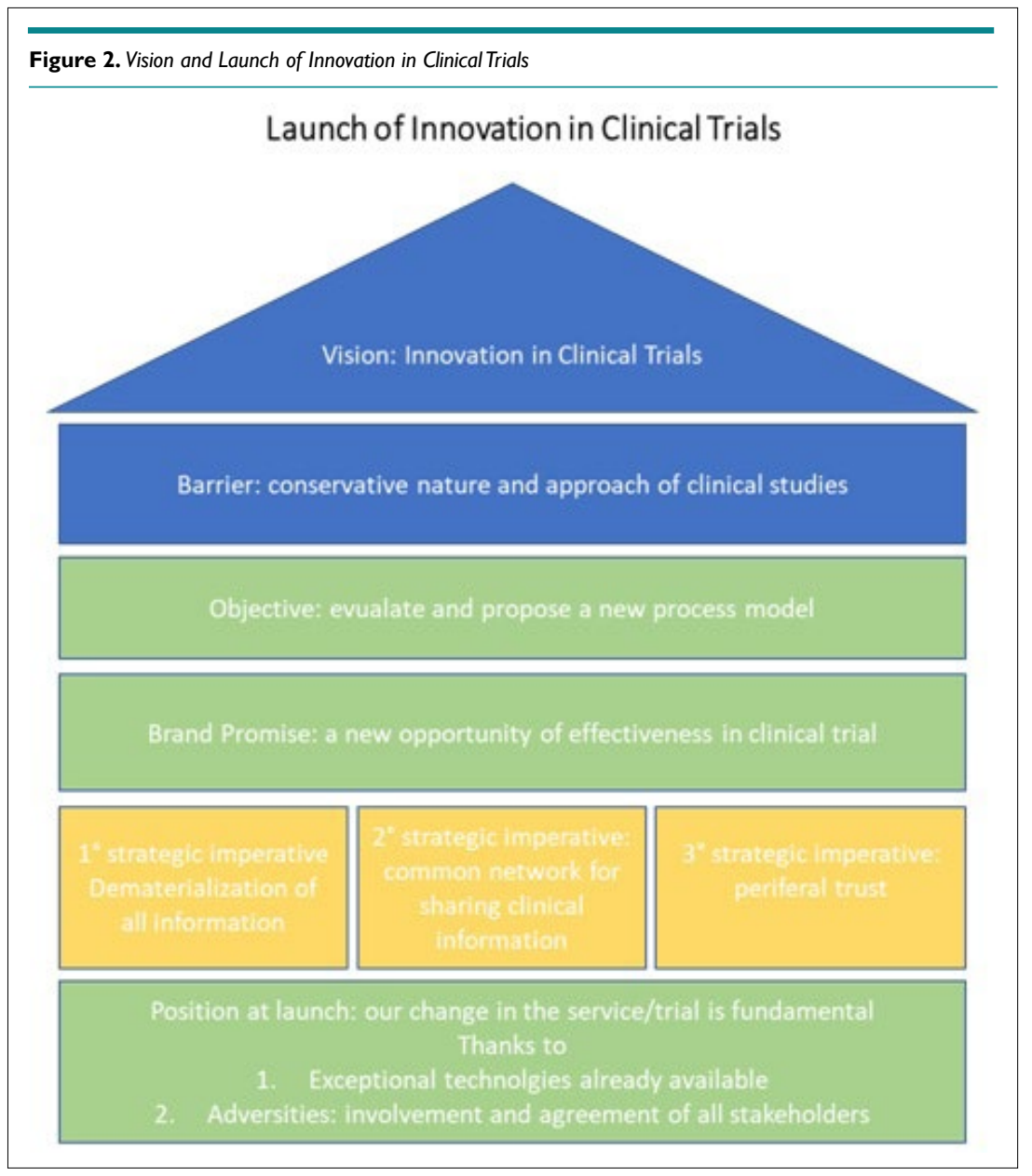

is about to be launched or identifying trends that are not easily recognizable in the current manual data analysis and revision process. ${ }^{9-11}$ Solutions performed by a specific program or algorithm, therefore allowing people to focus on either decisional activities or on tasks in which human intuition is a key success factor; more time dedicated to high value added activities and less effort wasted in mechanical data checks, then, in order to be able to improve the overall clinical studies quality, decrease their duration and lower their overall execution cost. This is for sure not the first time that the use of $\mathrm{AI}$ is mentioned in relation to clinical research, but how many companies have been brave enough to move the first steps towards a Proof of Concept (PoC)? And how many companies that have actually started a PoC have been able to complete one, overcoming the obstacles represented by the perfused fragmentation mentioned earlier?

The development of a technological solution is only one of the elements that are needed to carry out a true digital transformation in this field. ${ }^{12}$ To make this innovation as success, as further detailed in Figure 2, it is paramount that all the entities involved in the process: hospitals, companies and regulatory bodies are willing to pursue this objective and to recognize the actual need to change the process in order to make it more robust, efficient and secure. Continuing to work in the current way does not, per se, ensure a greater compliance or data integrity, but has the only effect to bound companies to an outdate clinical trial process vision that prevents innovation and discourages new ideas generation and implementation.

This field bears such a wealth of possibilities that starting the journey could be a strong driver for the creation of significant competitive advantage. Once a basic solution will be implemented it will be possible to further develop new tools that could allow to work on an early study approval based on solid data simulation and extrapolation that could be used when the need arise, such as in the COVID-19 emergency, without compromising the study quality or taking "shortcuts".

\section{WHO? |}

The COVID-19 emergency has partially contributed, from one side, to highlight how much clinical trial companies are still significantly unprepared to manage this kind of situations, and on the other, to impose on companies, hospitals and regulatory bodies a greater adoption, where possible and feasible in the given time frame, of new technologies.

This has been a true test bench for the companies' culture, often too based on the actual physical workplace and on the face-to-face relationship with customers and vendors, as well as for the resilience of their processes. 
The COVID-19 pandemic has been a great challenge for people and firms alike. It's not possible to overcome great trials without undergoing a change. It is therefore necessary to have the bravery to act a "re-adaptive surfing". Company leaders need to show the courage to innovate within their firms, they need to foster and "train" innovation amongst their people while being always ready to accept also completely different proposals and results, given the context.

Let's adopt a new perspective, then, that could allow us to expand our boundaries and to go exploring the unknowns where usually new opportunities lie, no matter if this changes the roadmaps that were defined maybe only a few months earlier.

Not only failing to adopt new and innovative approaches it's frustrating for the people that are committed to technological innovation, but new technologies that could significatively improve clinical studies have been already showcased and have fallen victim of an overcautious and stagnating environment. Will COVID-19 have the strength of not only changing the world but also changing the conservative and reactive approach that has characterized the clinical study sector for years?

Each great challenge requires a small amount of fear. It's the fear of stopping that should drive and increase the positive and innovative energy that already pervades and permeates companies worldwide.

\section{CONFLICTS OF INTEREST |}

The authors declare that they have no conflicts of interest.

\section{REFERENCES}

1. U.S. National Library of Medicine. clinicaltrials.gov Web site. https://clinicaltrials.gov/. Accessed June 10, 2020.

2. Kumar V. COVID-19 crisis: An update on the disruption of clinical trials. Preprints. 2020; 2020050411. doi: 10.20944/preprints202005.0411.v1

3. Grand View Research. Clinical Trials Market Size Worth $\$ 69.9$
Billion By 2027|CAGR: 5.1\%. grandviewresearch Web site. https://www.grandviewresearch.com/press-release/clinical-trials-market. Accessed June 10, 2020.

4. Freeman WR, Bartsch DU, Mueller AJ, Banker AS. Innovation and clinical trials. Arch Ophthalmol. 1999; 117(6): 846.

5. Marquis-Gravel G, Roe MT, Turakhia MP, Boden W, Temple R, Sharma A, et al. Technology-enabled clinical trials. Circulation. 2019; 140(17): 1426-1436. doi: 10.1161/CIRCULATIONAHA.119.040798

6. Sharma A, Harrington RA, McClellan MB, Turakhia MP, Eapen ZJ, Steinhubl S, et al. Using digital health technology to better generate evidence and deliver evidence-based care. J Am Coll Cardiol. 2018; 71(23): 2680-2690. doi: 10.1016/j.jacc.2018.03.523

7. Herndon JH, Hwang R, Bozic KH. Healthcare technology and technology assessment. Eur Spine J. 2007; 16(8): 1293-1302. doi: $10.1007 / \mathrm{s} 00586-007-0369-z$

8. Beyor N, Close K, Hakim N, Shapiro M, Ringel M, Smith B. A Digital Redesign for Clinical Trials. BCG Web site. https://www. bcg.com/it-it/publications/2019/digital-redesign-clinical-trials. aspx. Accessed June 10, 2020.

9. Google AI. Health Web site. https://research.google/teams/ health/. Accessed June 10, 2020.

10. Microsoft. Medical, health, and genomics. microsoft Web site. https://www.microsoft.com/en-us/research/research-area / medical-health-genomics $/$ ? facet $\% 5 \mathrm{Btax} \% 5 \mathrm{D} \% 5 \mathrm{Bmsr}$-research-area $\% 5 \mathrm{D} \% 5 \mathrm{~B} 0 \% 5 \mathrm{D}=13553 \&$ sort_by $=$ most-recent. Accessed June 10, 2020.

11. Amazon Web Services (AWS). Healthcare Providers \& Insurers in the Cloud. amazon Web site. https://aws.amazon.com/health/ providers-and-insurers/. Accessed June 10, 2020.

12. Sriram P. Remote consent clinical research. Clin Trial Pract Open J. 2019; 1(1): 39-41. doi: 10.17140/CTPOJ-1-109 